Irish Math. Soc. Bulletin

Number 84, Winter 2019, 51-55

ISSN 0791-5578

\title{
Solving cubic and quartic equations by radicals
}

\author{
C. T. C. WALL
}

\begin{abstract}
The rule for the solution of a cubic or quartic equation by radicals is obtained from elementary considerations of the geometry of the projective line.
\end{abstract}

\section{INTRODUCTION}

The formula $\frac{-b \pm \sqrt{ }\left(b^{2}-4 a c\right)}{2 a}$ for the roots of the quadratic equation $a x^{2}+b x+c=0$ is well known and easy to establish. The search for a corresponding formula for a cubic equation met with success in the 16th century, as can be found in one of the more colourful chapters of the history of mathematics. A method for solving quartic equations by radicals was also discovered in the 16th century, relatively soon after the solution of cubics, and other methods were then found.

Our object is neither to present the history nor the early versions of these arguments, but to give an account in the language of (projective) geometry to clarify the reasons for the formulae. A related version was given in [1].

\section{Cubic equations}

A cubic equation may be written as $a x^{3}+b x^{2}+c x+d=0$. We work over a field $K$ containing the coefficients $a, b, c, d$; to find a general formula, we may pick a field $k$ (my personal preference is the field $\mathbb{C}$ of complex numbers) and take $K$ as the pure transcendental extension $K=k(a, b, c, d)$, or just take $K=\mathbb{C}$; we will also discuss the case $K=\mathbb{R}$. Even the above rule for solving quadratic equations fails if $k$ has characteristic 2; for cubic and quartic equations we must further assume that $k$ does not have characteristic 2 or 3 .

To know what to expect, we refer to Galois Theory. As the group of the equation is the symmetric group $\mathfrak{S}_{3}$, to pass from $K$ to the root field of the cubic we need a quadratic extension (taking a square root), and a cubic extension which, provided $K$ contains a cube root of unity, involves taking a cube root.

From now on, we view the situation geometrically, so write the equation in homogeneous form as $h(x, y):=a x^{3}+3 b x^{2} y+3 c x y^{2}+d y^{3}$, and regard the root $\alpha$ as defining the point $P_{\alpha}=(\alpha: 1)$ on the projective line $P^{1}$, and correspondingly for the roots $\beta$ and $\gamma$. We assume that the roots are distinct.

A homography of $P^{1}$ is a map of the form $(x: y) \rightarrow(p x+q y: r x+s y)$; it is determined by the images of any 3 distinct points. Thus there is a unique $\phi: P^{1} \rightarrow P^{1}$ such that $\phi\left(P_{\alpha}\right)=P_{\beta}, \phi\left(P_{\beta}\right)=P_{\gamma}$ and $\phi\left(P_{\gamma}\right)=P_{\alpha}$; it follows that $\phi^{3}$ is the identity. This $\phi$ has just 2 fixed points, which we denote by $Q_{0}$ and $Q_{1}$.

The cubic $h(x, y)$ has Hessian $H(h):=h_{x x} h_{y y}-h_{x y}^{2}$, which is a quadratic covariant of $h$. Explicitly, if we change coordinates by $X=p x+q y, Y=r x+s y$ and $h(x, y)=$ $k(X, Y)$, we find that $h_{x x} h_{y y}-h_{x y}^{2}=(p s-q r)^{2}\left(k_{X X} k_{Y Y}-k_{X Y}^{2}\right)$.

2010 Mathematics Subject Classification. 12F10,14N05.

Key words and phrases. projective line, homography, involution, harmonic separation.

Received on 20-6-2019; revised 23-10-2019. 
The key observation is that the roots of $H(h)$ determine the points $Q_{0}, Q_{1} \in P^{1}$. To see this, we change coordinates in $P^{1}$ so that in the new coordinates $(X: Y)$, $Q_{0}=(0: 1)$ and $Q_{1}=(1: 0)$. Since $H(h)$ is a covariant, $H(k)$ is a multiple of $X Y$. Thus $\phi$ has the form $(X: Y) \rightarrow(\omega X: Y)$, where $\omega$ is a cube root of unity, the roots of $k$ may be written as $(\xi: 1),(\omega \xi: 1),\left(\omega^{2} \xi: 1\right)$, so

$$
k(X, Y)=a(X-\xi Y)(X-\omega \xi Y)\left(X-\omega^{2} \xi Y\right)=a\left(X^{3}-\xi^{3} Y^{3}\right),
$$

and $H(k)=-36 a^{2} \xi^{3} X Y$, which indeed has zeros at $Q_{0}$ and $Q_{1}$.

To solve the cubic $h$, we thus first factorise $H(h)$ in the form $(p x+q y)(r x+s y)$ (which involves solving a quadratic equation); then make the coordinate change $(X, Y)=$ $(p x+q y, r x+s y)$, which puts $h$ in the form $k(X, Y)=A X^{3}+D Y^{3}$. Then extracting the cube root of $-D / A$ allows us to factorise $k$, and changing coordinates back gives the desired result.

Returning to our original coordinates, we calculate:

$H(h)=36(a x+b y)(c x+d y)-(b x+c y)^{2}=36\left[\left(a c-b^{2}\right) x^{2}+(a d-b c) x y+\left(b d-c^{2}\right) y^{2}\right]$.

This quadratic (removing the factor 36) has discriminant

$$
\Delta(h):=(a d-b c)^{2}-4\left(a c-b^{2}\right)\left(b d-c^{2}\right)=a^{2} d^{2}+4 a c^{3}+4 b^{3} d-3 b^{2} c^{2}-6 a b c d,
$$

which coincides with the usual formula for the discriminant of $h$. We remark that in the case $a=1, b=0$ this formula reduces to $\Delta(h)=d^{2}+4 c^{3}$.

If the roots of $h$ are $\alpha, \beta, \gamma$, the quadratic extension is the one containing $\sigma:=$ $(\alpha-\beta)(\beta-\gamma)(\gamma-\alpha)$, since $\sigma^{2}$ is a symmetric function of $\alpha, \beta$ and $\gamma$. Since the vanishing of $\Delta$ is also the condition for $h$ to have equal roots, $\sigma^{2}$ agrees with $\Delta$ up to a scalar factor. To check the scalar, suppose $b=c=0$. Then $\sigma=(1-\omega)\left(\omega-\omega^{2}\right)\left(\omega^{2}-1\right) \alpha^{3}=3 \sqrt{ }-3 \alpha^{3}$, $\Delta=a^{2} d^{2}$ and $d=a \alpha^{3}$. Thus $a^{4} \sigma^{2}=-27 a^{4} \alpha^{6}=-27 a^{2} d^{2}=-27 \Delta$.

\section{Quartic equations I}

In this case, it is convenient to think of numbers as points on the conic $S_{0}$, with parametrisation $\left(t^{2}, t, 1\right)$ and equation $g_{0}=0$ in the plane $P^{2}$, where $g_{0}(x, y, z)=y^{2}-x z$. Write the quartic equation as $f(t)=0$, where

$$
f(t) \equiv a t^{4}+4 b t^{3}+6 c t^{2}+4 d t+e .
$$

The basic invariants of $f$ are the transvectant

$$
T(f)=a e-4 b d+3 c^{2}
$$

and the catalecticant

$$
K(f)=\left|\begin{array}{lll}
a & b & c \\
b & c & d \\
c & d & e
\end{array}\right| .
$$

The roots of $f$ correspond to the points of intersection of $S_{0}$ with the conic given by $g=0$, where

$$
g(x, y, z)=a x^{2}+4 b x y+c\left(4 y^{2}+2 x z\right)+4 d y z+e z^{2} .
$$

Here we can replace $g=0$ by any of the conics $S_{\lambda}$ given by $g-2 \lambda g_{0}=0$. The equation of $S_{\lambda}$ has matrix

and its determinant evaluates to

$$
\left(\begin{array}{ccc}
a & 2 b & c+\lambda \\
2 b+c & 4 c-2 \lambda & 2 d \\
c+\lambda & 2 d & e
\end{array}\right)
$$

$$
R(f):=2\left(\lambda^{3}-\lambda T(f)+2 K(f)\right) .
$$


$R(f)$ is called the cubic resolvent of $f$. If $\lambda$ takes a value making $R(f)$ vanish, the conic $S_{\lambda}$ is singular, and so breaks up into a pair of lines. Each of these lines meets $S_{0}$ in two points, and these points are the 4 points on $S_{0}$ giving the roots of $f$. Thus we have an arrangement of this set of 4 points in two pairs. Conversely, for each of the 3 such arrangements, the two lines each joining one of the pairs form a line-pair giving one of the singular conics $S_{\lambda}$.

The procedure for solving the quartic $f=0$ is now

(i) Choose a value of $\lambda$ such that $R(f)=0$.

(ii) Factorise $g-2 \lambda g_{0}$ as $\ell \ell^{\prime}$, where $\ell$ and $\ell^{\prime}$ are linear expressions.

(iii) Substitute $x=t^{2}, y=t, z=1$ in $\ell$, and solve the resulting quadratic equation; then do the same for $\ell^{\prime}$.

Step (i) involves the solution of a cubic, which we can achieve by taking a square root, then a cube root.

A short calculation shows that step (ii) also reduces to solving a quadratic, hence involves taking a square root; and step (iii) again involves solving a quadratic.

The first step in the solution involves taking the square root of the discriminant of the resolvent cubic, which is $\Delta(f):=\Delta(R(f))=T(f)^{3}-27 K(f)^{2}$. The quadruple of roots in $P^{1}$ is determined up to equivalence by the single invariant

$$
j(f):=\frac{T(f)^{3}}{T(f)^{3}-27 K(f)^{2}} .
$$

\section{Quartic equations II}

In the same style as our treatment of cubic equations, we consider involutions, i.e. homographies of $P^{1}$ of order 2 . Here it is convenient to use the inhomogeneous coordinate $t$ on $P^{1}$. An involution $I$ may be written as $a t t^{\prime}+b\left(t+t^{\prime}\right)+c=0$, or equivalently as $I(t)=-\frac{b t+c}{a t+b}$. $I$ has 2 fixed points on $P^{1}$ and is determined by them. Two points are paired by $I$ if and only if they harmonically separate the fixed points. An involution $I^{\prime}$ commuting with $I$ must either preserve the fixed points of $I$ (in which case it coincides with $I$ ) or interchange them.

The four roots of a quartic equation $f=0$ determine, as above, four points of $P^{1}$. For each arrangement of these four points in two pairs, say $(\alpha, \beta)(\gamma, \delta)$ there is a unique involution of $P^{1}$ interchanging the pairs $(\alpha, \beta)$ and $(\gamma, \delta)$. These involutions correspond to the singular conics $S_{\lambda}$ just described: the above involution corresponds to the conic which is the union of the lines $P_{\alpha} P_{\beta}$ and $P_{\gamma} P_{\delta}$. The 3 such arrangements yield 3 involutions, which form a group isomorphic to the four group. Since any two of the involutions commute, the corresponding pairs of fixed points separate harmonically, so the 6 fixed points (which can be found as the zeros of the jacobian of $f$ with its Hessian $H(f)$ ) form the vertices of a regular octahedron under a suitable identification of $P^{1}(\mathbb{C})$ with the 2 -sphere. The symmetry group of the octahedron is $\mathfrak{S}_{4}$, isomorphic to the group of $f$.

The calculations simplify if $I$ takes the form $I(t)=-t$. To achieve this, choose a root $\lambda$ of the resolvent cubic: then the quadratic $S_{\lambda}$ is a line pair. The point $P$ of intersection of these lines can be found by solving the linear equations

$$
\partial\left(g-2 \lambda g_{0}\right) / \partial x=\partial\left(g-2 \lambda g_{0}\right) / \partial y=\partial\left(g-2 \lambda g_{0}\right) / \partial z=0
$$

(that these are consistent follows since $\lambda$ is a root of $R$ ). The corresponding involution is cut on $S_{0}$ by lines through $P$. If $P$ has coordinates $\left(x_{0}, y_{0}, z_{0}\right)$, then the points with parameters $t$ and $t^{\prime}$ lie on a line through $P$ if the determinant

$$
\left|\begin{array}{ccc}
x_{0} & y_{0} & z_{0} \\
t^{2} & t & 1 \\
t^{\prime 2} & t^{\prime} & 1
\end{array}\right|
$$


vanishes, or equivalently, removing the factor $\left(t-t^{\prime}\right)$, if

$$
x_{0}-y_{0}\left(t+t^{\prime}\right)+z_{0} t t^{\prime}=0 .
$$

Let $\xi$ be a solution of $\xi^{2} x_{0}+2 \xi y_{0}+z_{0}=0$, and set $w=\frac{\xi t+1}{z_{0} t / \xi+x_{0}}$; then indeed the involution takes the form $I(w)=-w$, and now taking $w$ as coordinate reduces $f$ to the form $a w^{4}+6 c w^{2}+e$. In this situation, the procedure for solving $f=0$ reduces to first solving the quadratic equation for $w^{2}$, and then taking square roots of the solutions.

When $b=d=0$, the invariants reduce to $T(f)=a e+3 c^{2}$ and $K(f)=c\left(a e-c^{2}\right)$, and $R(f)$ factorises as $R(g)=2(\lambda-2 c)\left(\lambda^{2}+2 c \lambda+c^{2}-a e\right)$; the root $\lambda=2 c$ corresponds to the chosen involution.

\section{Over THE REAL NUMBERS}

For a cubic equation $h=0$, if $\Delta(h)<0$, the quadratic equation $H(h)$ has real roots, we can reduce the Hessian $H(h)$ to $x y$, and then require the 3 cube roots of a real number, so only one of the roots of $h$ is real.

If however $\Delta(h)>0$, the quadratic has conjugate complex roots, and we can reduce $H(h)$ to $x^{2}+y^{2}$. Geometrically, the Hessian points are now $( \pm i: 1)$ and $\phi$ is a real rotation through $2 \pi / 3$. In this case all 3 roots of $h$ are real.

For a quartic equation, at each stage of the above procedure where the square root of an expression $E$ is taken, there are two cases according to the sign of $E$; this seems to lead to huge numbers of cases. However there are just 3 cases for the quartic, according as it has 0,2 or 4 real roots.

If $f$ has 4 real roots $p, q, r, s$, the resolvent cubic has 3 real roots, corresponding to the arrangements of the roots in pairs as $(p, q)(r, s),(p, r)(q, s)$ and $(p, s)(q, r)$. In each of these cases, the conic $S_{\lambda}$ consists of 2 real lines, and each of these lines meets $S_{0}$ in 2 real points.

If $f$ has 2 real roots $p, q$ and a conjugate complex pair $z, \bar{z}$, then the conics corresponding to the arrangements $(p, z)(q, \bar{z})$ and $(p, \bar{z})(q, z)$ are conjugate to each other, so $R$ has just 1 real root. For the arrangement $(p, q)(z, \bar{z})$ we have 2 real lines, with one line meeting $S_{0}$ in 2 real points, the other in none.

If $f$ has 0 real roots, the roots form 2 conjugate complex pairs $(w, \bar{w})$ and $(z, \bar{z})$, and the conic corresponding to the arrangement $(w, \bar{w})(z, \bar{z})$ consists of 2 real lines, neither having a real point of intersection with $S_{0}$, while each of the conics corresponding to $(w, z)(\bar{w}, \bar{z})$ and $(w, \bar{z})(z, \bar{w})$ is real, but consists of a pair of conjugate complex lines. Here again $R$ has 3 real roots.

We have seen that the sign of the discriminant determines whether $R$ has 1 or 3 real roots. If it has 3 , deciding whether $f$ has 0 or 4 real roots is less simple, but it can be shown that $f$ has 4 real roots if and only if both $a c-b^{2}$ and $a^{3} e-4 a^{2} b d-9 a^{2} c^{2}+$ $24 a b^{2} c-12 b^{4}$ are negative.

We can rewrite the above in terms of involutions, following [2]. Write $J$ for complex conjugation on $P^{1}$ : then an involution $I$ is real, i.e. has real coefficients, if and only if $J I=I J$. If $I$ is a real involution, then either

type $(\mathrm{r})$ : its fixed points are both real (an example is $I_{0}(t)=-t$ ); or

type (c): its fixed points are complex conjugates (an example is $I_{i}(t)=-t^{-1}$ ).

If $f$ is a real quartic, the map $J$ must preserve the octahedron $O$ formed by the fixed points of the 3 involutions. There are two cases:

(a) $J$ interchanges a pair of opposite vertices of $O$ and fixes the other vertices;

(b) $J$ fixes one pair of opposite vertices and interchanges the other two pairs. 
In case (a), we can take the vertices as $(0, \infty)( \pm 1)( \pm i)$ (with $J$ the usual complex conjugation); the involutions are then $t \rightarrow-t, t \rightarrow 1 / t, t \rightarrow-1 / t$; all are real, the first two of type (r), the other of type (c).

In case (b), we can take the involutions as $t \rightarrow-t, t \rightarrow i / t, t \rightarrow-i / t$; the first is real of type (r), the other two are complex conjugates; and the vertices are $(0, \infty)\left( \pm e^{i \pi / 4}\right)\left( \pm e^{-i \pi / 4}\right)$.

In each case there is at least one real involution of type $(\mathrm{r})$ preserving $f$.

Conversely, given a real involution $I$ preserving $f$, we seek to follow the above procedure for reducing $I$ to the form $t \rightarrow-t$. First we solve linear equations (so can work over $\mathbb{R}$ ), to find a point $P_{0}$ with coordinates $\left(x_{0}, y_{0}, z_{0}\right)$. We then require the square root of $y_{0}^{2}-x_{0} z_{0}$. The sign of $y_{0}^{2}-x_{0} z_{0}$ depends whether $P_{0}$ is inside or outside the conic $S_{0}$, hence on whether the involution $I$ has 0 or 2 real fixed points. We can thus reduce $I$ to $t \rightarrow-t$ provided $I$ has type (r).

In case (a), the roots of $f$ have the form $\pm \alpha, \pm \alpha^{-1}$, so $f(z)=\left(z^{2}-\alpha^{2}\right)\left(z^{2}-\alpha^{-2}\right)=$ $z^{4}+6 c z^{2}+1$, with $-6 c=\alpha^{2}+\alpha^{-2}$. There are 3 cases:

(i) $\left(c<-\frac{1}{3}\right)$ all roots are on the real axis,

(ii) $\left(c>\frac{1}{3}\right)$ all roots on the imaginary axis,

(iii) $\left(|c|<\frac{1}{3}\right)$ all roots on the unit circle.

However we could also have begun with the other real involution of type (r). The involutions $t \rightarrow-t$ and $t \rightarrow 1 / t$ are interchanged by the substitution $u=\frac{t+1}{t-1}$; making this change replaces $c$ by $\frac{1-c}{1+3 c}$, and interchanges cases (ii) and (iii).

In case (a), we have $\Delta=T^{3}-27 K^{2}=\left(1-9 c^{2}\right)^{2}>0$, and $j=\frac{T^{3}}{T^{3}-27 K^{2}}=\frac{\left(1+3 c^{2}\right)^{3}}{\left(1-9 c^{2}\right)^{2}}$, which is $>1$. If $c= \pm \frac{1}{3}, j=\infty$, if $c= \pm 1, j=1$; if $c=0, j=1$. In each of the cases (i)-(iii), $j$ can take any value $>1$; so $j$ is of no use to distinguish these cases.

In case (b), the roots have the form $\pm \alpha, \pm i / \alpha$; two are real and two are complex conjugate, and $f$ takes the form $t^{4}+6 c t^{2}-1$. Here $T=3 c^{2}-1, K=-c\left(1+c^{2}\right)$, and $\Delta=-\left(1+9 c^{2}\right)^{2}<0$.

\section{REFERENCES}

[1] W. M. Faucette, A geometric interpretation of the solution of the general quartic polynomial, Amer. Math. Monthly 103(1) (1996), 51-57.

[2] C. T. C. Wall, Pencils of real binary cubics, Math. Proc. Camb. Phil. Soc. 93 (1983), 477-484.

C.T.C.Wall took his Ph.D. in Cambridge in 1960, and held a teaching post at the University of Liverpool from 1965-1999, except from 1983-88 when he held an EPSRC Senior Fellowship. His research has mainly been in differential topology and singularity theory

School of Mathematical Sciences, University of Liverpool.

E-mail address: ctcw@liverpool.ac.uk 